\title{
Lattice-matched II-VI/III-V Materials and Virtual Substrates for Multijunction Solar-Cell Applications
}

\author{
L. Ouyang, ${ }^{*}$ X.-B. Zhang, ${ }^{* *}$ S. Wang, ${ }^{* * *}$ X. Lu, ${ }^{* * *}$ Y.-H. Zhang, ${ }^{* * *}$ X. Liu, ${ }^{* * * *}$ J. Furdyna, ${ }^{* * * *}$ \\ and D. J. Smith* \\ * Department of Physics, Arizona State University, Tempe, AZ 85287 \\ ** School of Materials, Arizona State University, Tempe, AZ 85287 \\ *** Center for Nanophotonics, Arizona State University, Tempe, AZ 85287 \\ **** Department of Physics, University of Notre Dame, Notre Dame, IN 46556
}

There is much current interest in strategies to increase solar-cell efficiency by taking advantage of a greater portion of the solar energy spectrum. One obvious approach is to use a multijunction design based on semiconductor materials with band gaps that span a broad spectral range. The monolithic integration of II-VI (MgZnCd, SeTe) and III-V (AlGaIn, AsSb) alloys lattice-matched to the socalled 6.1- $\AA$ substrates of either GaSb or InAs is one possible option for achieving this objective [1]. This paper compares the microstructure of ZnTe epilayers grown by molecular beam epitaxy on $\mathrm{GaSb}(100)$, InAs(100) and $\mathrm{Si}(211)$ substrates. Samples were prepared for cross-section observation using standard techniques, but with the sample held at liquid-nitrogen temperature during ionmilling to minimize any milling artifacts. Most images were recorded with a JEM-4000EX highresolution electron microscope operated at $400-\mathrm{keV}$ with a structural resolution of $\sim 1.7 \AA$.

Cross-sectional electron micrographs of the $\mathrm{ZnTe} / \mathrm{GaSb}$ sample revealed highly coherent interfaces with very low defect density, as shown by the example in Fig.1(a). Higher magnification views of the $\mathrm{ZnTe} / \mathrm{GaSb}$ interface revealed the absence of misfit dislocations, as might be expected given the very small mismatch $(\sim 0.13 \%)$ between the two materials. Moreover, even the precise position of the interface was extremely difficult to pinpoint in high-resolution images, which was attributed to the closely similar average atomic numbers of the two materials. It was also noteworthy that there was no sign of any interfacial phases in the vicinity of the interface. In the case of the ZnTe/InAs sample, some small residual strain was visible at the interface, presumably because of the slightly larger lattice mismatch. Nevertheless, as should be apparent from Fig. 2, there was again a marked absence of any interfacial misfit dislocations. Thin ZnTe films grown on bulk Si substrates have been previously used as nucleation layers for growth of $(\mathrm{Cd}, \mathrm{Zn}) \mathrm{Te}$ epilayers, which could in turn serve as virtual substrates for subsequent deposition of lattice-matched II-VI/III-V materials. Figure 3 shows a thick ZnTe layer grown directly on a Si(211) substrate. Well-separated \{111\}-type stacking faults are visible propagating through to the ZnTe surface in Fig. 3(a). The higher magnification image in Fig. 3(b) shows that much of the misfit strain between these two semiconductors is relieved at the interface, without significant generation of dislocations in the epitaxial layer. Thus, this ZnTe/Si combination could be considered as a prmising virtual substrate for future deposition of II-VI/III-V alloy combinations.

\section{References}

[1] S. Wang, et al., J. Cryst Growth 311 (2008) 2116.

[2] X.-B. Zhang, et al., J. Electronic Materials 38 (2009) 1558.

[3] This work is primarily supported by the Science Foundation Arizona Contract No. SRG-019007.. The authors acknowledge the use of facilities in the John M. Cowley Center for High Resolution Electron Microscopy at Arizona State University. 


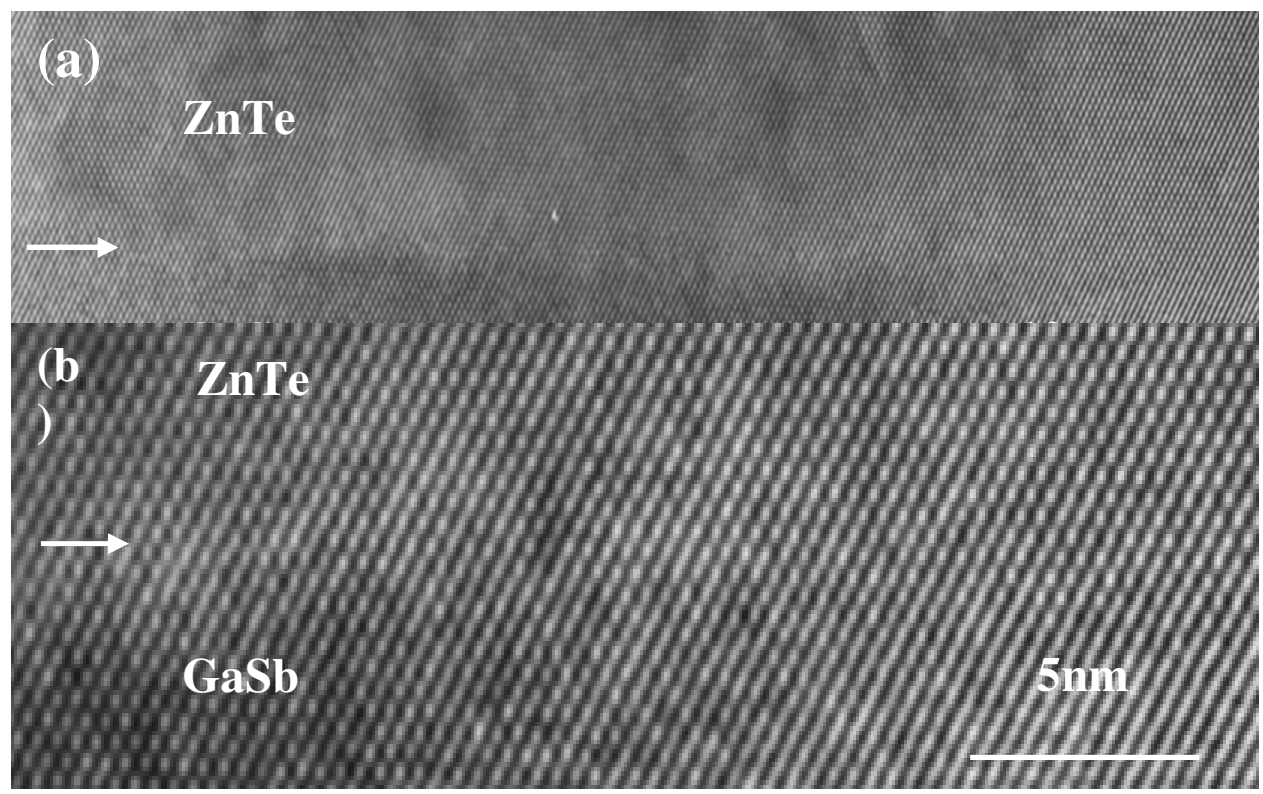

FIG. 1. (a) Cross-sectional electron micrograph of highly coherent ZnTe/GaSb interface showing absence of interfacial misfit dislocations; (b) Higher magnification image.

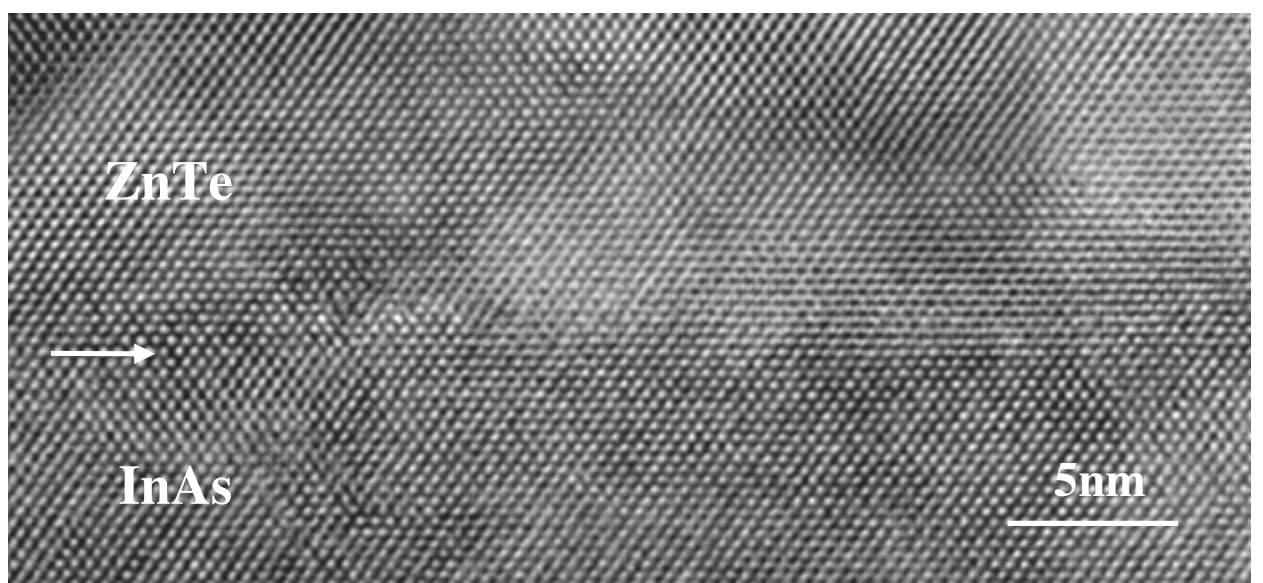

FIG. 2. Cross-sectional electron micrograph showing highly coherent ZnTe/InAs interface.
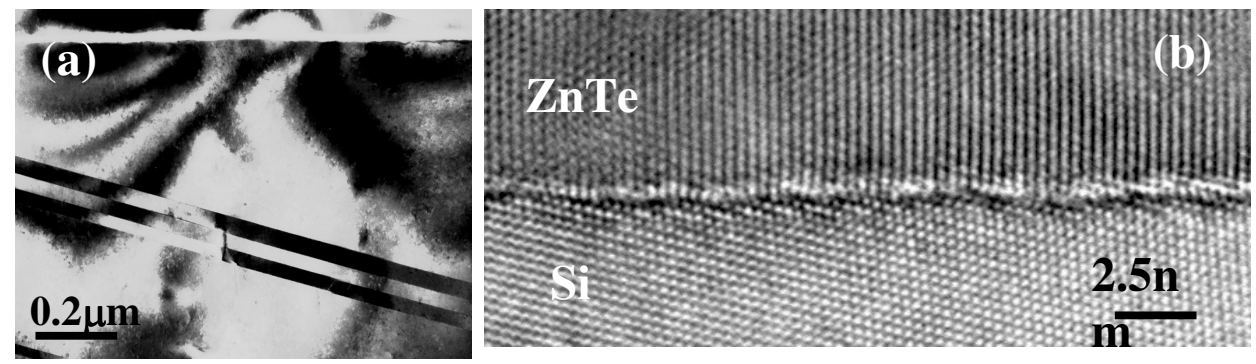

FIG. 3. Cross-sectional electron micrographs of thick ZnTe/Si $\{211\}$ sample: (a) Low magnification view taken from region near top surface showing well separated $\{111\}$-type stacking faults; (b) high-resolution lattice-fringe image showing enlarged view of $\mathrm{ZnTe} / \mathrm{Si}$ interface region. 